ISSN: 2641-9475

\title{
Tumor-infiltrating lymphocytes expression in stage IIIc/IV of high-grade serous ovarian cancer: Variation with neoadjuvant chemotherapy and prognostic value
}

\author{
K Rojas' ${ }^{1}$, G Prado-Vazquez², C Barcena ${ }^{3}$, L Trilla-Fuertes², A Gamez-Pozo ${ }^{2,4}$, A Zapater-Moros ${ }^{4}$, \\ M Ferrer-Gomez ${ }^{4}$, L Lema ${ }^{1}$, R Garcia-Martin ${ }^{3}$, A Maroto $^{3}$, JL Rodriguez ${ }^{3}$, C Mendiola ${ }^{1}$, JA Fresno \\ Vara $^{4}$, L Paz-Ares $^{1}$ \\ ${ }^{1}$ Department of Medical Oncology, Hospital 12 de Octubre, Madrid, Spain. \\ ${ }^{2}$ Biomedica Molecular Medicine SL, Madrid, Spain. \\ ${ }^{3}$ Department of Pathology, Hospital 12 de Octubre, Madrid, Spain. \\ ${ }^{4}$ Molecular Oncology \& Pathology Lab, Institute of Medical and Molecular Genetics-INGEMM, Hospital Univer- \\ sitario La Paz, Madrid, Spain.
}

\section{${ }^{*}$ Corresponding author:}

\section{Katerin Ingrid Rojas Laimito}

Department of Medical Oncology,

Hospital 12 de Octubre,

Av. Cordoba, s/n, 28041 Madrid, Spain

Received : January 23, 2019

Published : March 14, 2019

\section{ABSTRACT}

Background: Ovarian cancer is a malignancy with a complex immune suppressive microenvironment mediated by the recruitment or induction of cluster differentiation factor $4+(C D 4+)$ regulatory T cells. The purpose of this study was to assess the effect of neoadjuvant chemotherapy (NACT) on immune activation in stage IIIc/IV of High-Grade Serous Ovarian Carcinoma (HGSC), and its relationship to treatment response.

Patients and Methods: We retrospectively identified 33 patients diagnosed with HGSC and treated with neoadjuvant platinum-paclitaxel from 2005-2014. Pre- and post-neoadjuvant treatment tissue samples were submitted to immunohistochemical analyses with anti-CD3, CD4 and CD8 antibodies for the identification of tumor-infiltrating lymphocytes (TILs). The staining results were analyzed and were blindly evaluated with respect to clinical features. Pathological response classification to NACT was made according to Steffen Bohm.

Results: The mean age of patients was 63.44 years (46.5384.14). Germline BRCA1/2-mutation status was negative in $58.82 \%$ of patients (10/17); BRCA1/2-mutation was positive in $11.76 \%$ ( 2/17); and a variant of uncertain significance was found in $29.41 \%(5 / 17)$. The majority of patients (78.8\%) were stage IIIc. The area under the ROC curve of post-surgery TILs for complete pathological response was: CD4 (epithelial): [0.73(0.5; 0.97), p: 0.084]; CD4 (stromal): [0.74(0.51; 0.97), p: $0.077]$ and CD8 (epithelial): [0.81 (0.63; 1.0), p: 0.02]. The expression of epithelial CD4 TILs in pre-surgery samples $(\leq 0.5[\mathrm{OR}$ : $0.7(0.01 ; 0.86), \mathrm{p:} 0.038])$ and epithelial CD8 TILs in post-surgery samples ( $\leq 5.4[\mathrm{OR}: 0.1(0.01 ; 1.19, \mathrm{p}: 0.06])$ proved to be a marker of good prognosis for pathological response. Survival 
analysis demonstrated that the expression of epithelial CD3 $\leq$ 4, 3 in pre-surgery samples is a marker of poor prognosis.

Conclusion: The high number of tumor-infiltrating lymphocytes in post-surgery samples was significantly associated with higher rates of complete pathological response and bet-

\section{INTRODUCTION:}

Epithelial ovarian cancer (EOC) is the most lethal gynecological tumor. Ovarian cancer $(\mathrm{OC})$ is the fifth most common type cause of cancer in women [1] and the fourth most common cause of cancer death in women [2]. High-grade serous carcinoma (HGSC) comprises approximately $70 \%$ of all EOC and accounts for the majority of deaths. HGSC is an aggressive neoplasms, presents in advanced stages (III-IV) and has a high frequency of TP53 mutations [3, 4]. This suggests that there may be other tumor microenvironment or host characteristics with a dominant role in survival.

To date, the standard therapy for advanced ovarian cancer is cytoreductive surgery or debulking surgery, followed by chemotherapy with the combination of carboplatin (area under the curve (AUC), 5.0-7.5) and paclitaxel $175 \mathrm{mg} / \mathrm{m}^{2}$ every 3 weeks, for 6 cycles)[5] or three cycles of platinumbased neoadjuvant (NACT), followed by interval debulking surgery, and then at least three more cycles of platinum-based chemotherapy[6].

In recent years, there has been interest in understanding the role of immune response. Ovarian cancer is an immune reactive malignancy with a complex immune suppressive network that blunts successful immune eradication. This suppressive microenvironment may be mediated by recruitment or induction of CD4+ regulatory T cell (Tregs)[7].

A number of studies have demonstrated that the presence of tumor-infiltrating lymphocytes (TIL) in surgically resected tumor tissue correlates with a favorable prognosis, especially in HGSC. Landmark study by Coukos et al. , retrospective analysis of 186 stage III-IV EOC patients revealed that the 5 -year overall survival (OS) rate was 38\% for patients with CD3+ TIL-positive tumors, compared with only $4.5 \%$ for CD3+ TIL-negative tumors $[8,9]$. Other studies revealed that the CD8+ subset of TIL correlates more strongly with survival [10-13].

Tregs are a heterogeneous CD4+ T cell subpopulation whose primary function is to immunoregulate by blocking the function of activated T cells. CD4+ Tregs can be divided into ter prognosis. It is convenient to carry out further and multicentric studies to validate these results.

KEYWORDS: tumor-infiltrating lymphocytes, ovarian cancer, neo-adjuvant chemotherapy.

two main subsets: naturally occurring Tregs with a CD4+CD25+ forkhead box P3 (FOXP3)+ phenotype and induced Tregs with a variable CD25 expression [14]. Curiel et al. initially showed a strong association of CD4+CD25+ T cell with poor survival [15].

The expression of PD-L1 in a range of malignancies including renal, esophageal and colorectal cancers, suggesting that some tumors have evolved high levels of expression of this molecule, possibly suppressing anti-tumor $T$ cell responses[16,17]. As a result, PD-L1 offers potential as a prognostic marker as well as being a target for therapy.

Hamanishi et al. studied expression of PD-L1 and PD-L2 on ovarian tumors of different grades and found a significantly worse overall survival in patients whose tumor expressed one or both of these ligands. They also showed an inverse correlation between PD-L1 expression and intraepithelial CD8 count, a known prognostic marker[18-20].

The discovery of the importance of the immune system in the development of cancer can help us in the determination of new diagnostic and prognostic parameters and the possibility of using serum markers instead of biopsies. This evades the possibility of non-representative biopsies, intermetastasesheterogeneity and might be able to avoid invasive diagnostic techniques[21,22].

The purpose of this study was to assess the variation of neoadjuvant chemotherapy on tumor-infiltrating lymphocytes expression in stage IIIc/IV of high-grade serous ovarian carcinoma, and prognostic value.

\section{MATERIALS AND METHODS:}

\section{Patients and samples}

The person directing the project identified those patients whose diagnosis was proven to be HGSC, stage III-IV (FIGO), underwent primary debulking surgery (PDS) and postchemotherapy residual disease and requested them to participate in the study. 
The study design will be Descriptive Observational: Population -correlational (relationship between CD3+, CD4+, and CD8+

$T$ Cells on the tumor tissue obtained from a diagnostic laparoscopy or diagnostic core biopsy and post-chemotherapy residual disease in HGSC. Patients were treated at Hospital 12 de Octubre (Madrid-Spain) between 2005 and 2014.

Pathological response classification to NACT was made according to Steffen Bohm (JCO 2015). Score response system (CRS) was explicitly defined (CRS-1; No or minimal tumor response, CRS-2; Appreciable tumor response amid viable tumor that is readily identifiable, CRS-3; Complete or nearcomplete response).

The selected tumor samples, formalin-fixed paraffinembedded tissue (FFPE), were retrieved from the archive of the Hospital 12 de Octubre -Pathology Department. The cases were selected based on the histological type, stage and follow-up.

The study was approved by the ethical committee of our institution. All patients included in the study gave written informed consent for research use of tissue specimens.

\section{Formalin-fixed paraffin embedded tumor biopsies}

Pre-treatment omental or peritoneal biopsies were obtained at diagnostic laparoscopy or diagnostic core biopsy. Sections of 4-um thickness were mounted on glass slides. Blocks of omentum removed at interval debulking surgery (IDS) after NACT, were reviewed by a pathologist according to the Recommendations from the International Collaboration on Cancer Reporting (ICCR guidelines). Sections of samples that representing the area of the tumor with worst response were selected.

\section{Fresh tumor biopsies}

Omental biopsies were collected in the operating room from untreated patients undergoing PDS or diagnostic laparoscopy and from patients undergoing IDS after NACT and fresh-frozen samples were histologically matched to FFPE specimens of the same patient by hematoxylin and eosin review.

\section{Immunohistochemistry}

Immunohistochemistry was performed on $4 \mu \mathrm{m}$-thick sections of formalin-fixed, paraffin-embedded tissue samples using anti-CD3 (LN10, Leica), CD4 (4B12, Leica), CD8 (C8/144B, Dako). Immunostaining was performed on a Leica Bond-III stainer (Leica Biosystem, Newcastle, UK) for anti-CD3 staining (1/150 dilution). Immunostaining was also performed on Dako stainer for anti-CD4 (1/10 dilution) and anti-CD8 (1/10 dilution).

The staining results were analyzed by one pathologist and were blindly evaluated with respect to clinical features. The slides were scored as positive for strong and weak staining intensity, negative in the absence of staining or not applicable (NA) to indicate insufficient material for analysis.

\section{Statistical analysis}

Mann-Whithney tests were done to compare the percentage of infiltrated lymphocytes between the sample before and the sample after chemotherapy. Analyses were done using GraphPad Prism. We make response prediction models with infiltration percentages but no significant results have been obtained.

Values of CD3+, CD4+ and CD8+ TL as general predictors of pathological response ( $\mathrm{CCR}$ ) were performed using an ROC curve and the confidence interval $(95 \% \mathrm{Cl})$ of the area under the curve; in the case that was significant an approximate cutoff point was made.

The risk estimate for complete pathological response of TILS was calculated using the Odds-ratio (OR) and for the overall survival (OS), the estimated Hazard Ratios (HR) with their $95 \% \mathrm{Cls}$ were estimated. In all cases a type I error of $0.05 \%$ is considered.

\section{RESULTS}

\section{Patient's characteristics}

We retrospectively identified 33 patients diagnosed with HGSC and treated with neoadjuvant platinum-paclitaxel from $2005-2014$.

The mean age of patients was 63.44 years (46.53-84.14). Germline BRCA $1 / 2$ - mutation status was negative in $58.82 \%$ of patients (10/17); BRCA 1/2 - mutation was positive in 11.76 $\%(2 / 17)$; and variant of uncertain significance was found in $29.41 \%(5 / 17)$. The majority of patients (78.8\%) were stage IIIC (Table 1). 
Table 1: Clinic, histology and genomic characteristics.

\begin{tabular}{|c|c|}
\hline $\begin{array}{c}\text { Age, years } \\
\text { Median }\end{array}$ & 63.44 \\
Range & $46.53-84.14$ \\
\hline Histological type & High-grade serous carcinoma \\
\hline FIGO stage: & \\
IIIC & $26 / 33(78.8 \%)$ \\
IV & $7 / 33(21.2 \%)$ \\
\hline Germline BRCA 1/2: & $10 / 17(58.82 \%)$ \\
Negative & $2 / 17(11.76 \%)$ \\
Positive & $5 / 17(29.41 \%)$ \\
\hline
\end{tabular}

The area under the ROC curve of post-surgery TILs for complete pathological response were: CD4 (epithelial): [0.73(0.5; 0.97), p: 0.084]; CD4 (stromal): [0.74(0.51; 0.97), p: 0.077] and CD8 (epithelial): $[0.81(0.63 ; 1.0), p: 0.02]$. The expression of epithelial CD4 TILs in pre-surgery samples ( $\leq 0.5[\mathrm{OR}: 0.7(0.01$; 0.86), p: 0.038]) and epithelial CD8 TILs in post-surgery samples ( $\leq 5.4$ [OR: $0.1(0.01 ; 1.19, \mathrm{p}: 0.06])$ proved to be a marker of good prognosis for pathological response. Survival analysis demonstrated that the expression of epithelial CD3 $\leq 4,3$ in pre-surgery samples is a marker of poor prognosis. Figures 1 , 2 and 3.

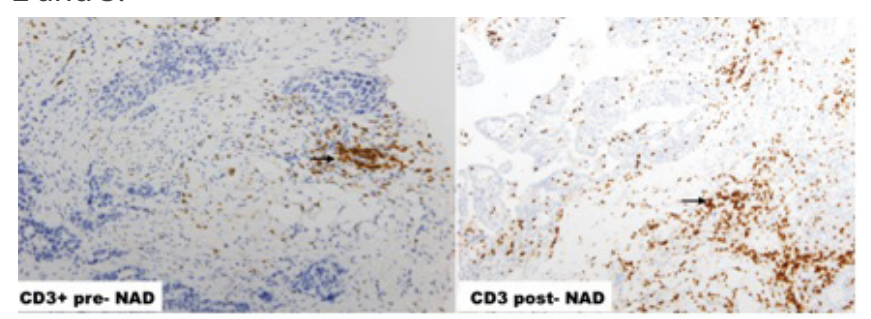

Figure 1: CD3+TILs increased significantly post-NACT.

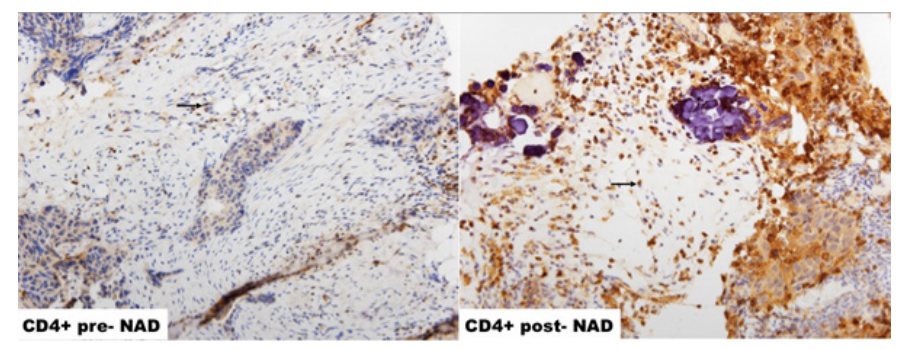

Figure 2: CD4+ TILs increased significantly post-NACT.

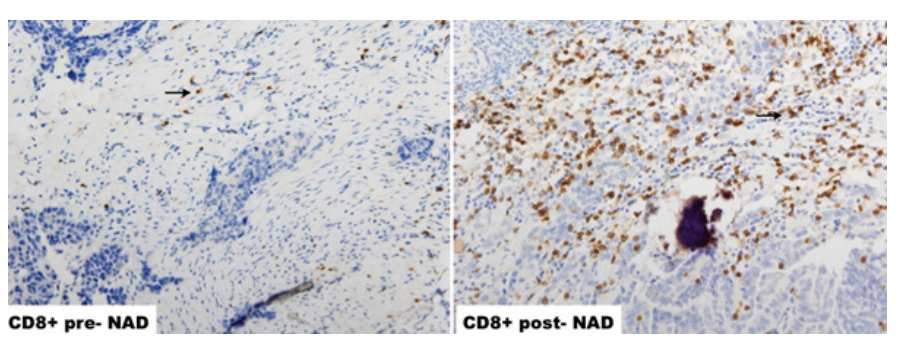

Figure 3: CD8+ TILs increased significantly post-NACT.

Mann-Whitney test was done to compare the percentage of infiltrated lymphocytes in the samples before and after receiving the neoadjuvant chemotherapy. There were significant differences between the percentage of both epithelial and stromal CD8 and in the percentage of epithelial CD3 (Figure 4).

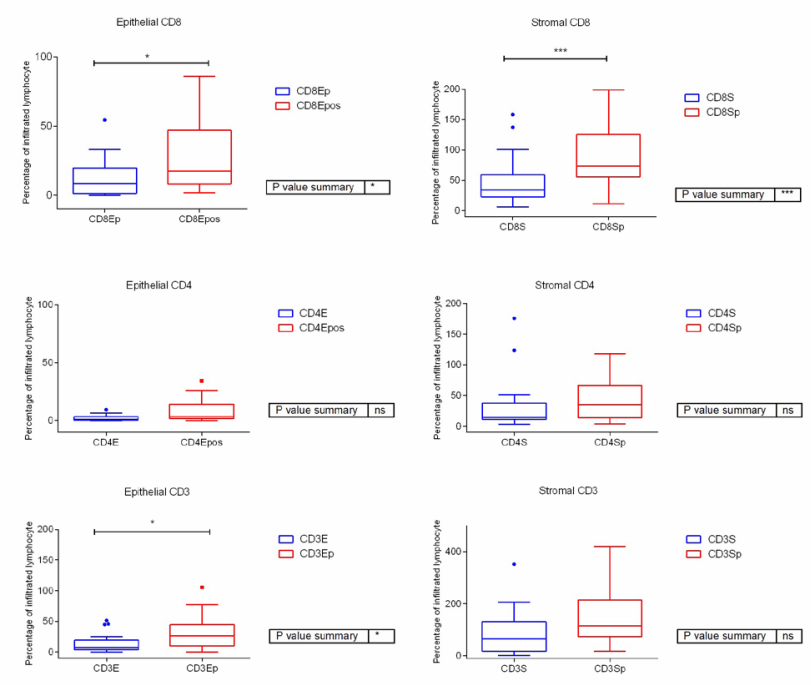

Figure 4: Percentage of lymphocyte infiltration in the pre and the post chemotherapy samples. ( $p=$ sample after neoadjuvant treatment)

\section{DISCUSSION}

The tumor biology, inflammatory cells, including lymphocytes, extracellular matrix and a rich cytokine environment contribute in concert to a microenvironment, that fosters inflammation, which paradoxically drives tumor growth, angiogenesis and promotes tumor infiltration and metastasis, while simultaneously inhibiting anti-tumor immunity.

A spontaneous antitumor immune response has been convincingly demonstrated in some patients with ovarian cancer. Tumor-reactive T cells and antibodies have been detected in peripheral blood, tumors or ascites of patients with advanced stage disease at diagnosis. Importantly, the detection of antitumor immune response in the form of intraepithelial (also called intratumoral) tumor-infiltrating lymphocytes (TILs; i.e. T 
cells infiltrating tumor islets) predicts significantly longer survival in ovarian cancer[23-25].

We demonstrate that the effect of NACT on a human metastatic tumor microenvironment induces activation of $\mathrm{CD} 3+\mathrm{T}$ cells, CD4+ T cells and CD8+ T cells. Importantly, even those patients who had a poor response to three cycles of platinum-based chemotherapy had a high number of CD8+ T cells. Our findings are supported by a previous study reporting an association of CD8+ T cells and tumor eradication and concurrent activation CD4+ T cells is required to support potent cytotoxic T lymphocytes[26-31]. A high number of tumor infiltrating cytotoxic $T$ lymphocytes was associated with a better overall survival, presumably due to the stronger effect in non-optimally debulked patients[32].

Zhang et al. recently showed that, in epithelial ovarian cancer, the presence of intratumoral CD3+ T cells was associated with improved survival. Other study executed by Curiel et al., reported that tumor infiltration by a subpopulation of CD3+ CD4+ T cells with immunosuppressive properties predicted reduced survival in EOC[8,10,15].

Despite the existence of controversial published data, the results are in favor of the fact that the increase in the T lymphocyte population is associated with greater survival.

The impact of intraepithelial CD3+ or CD8+ T cells was confirmed by multiple independent studies on ethnically and geographically diverse populations. Intraepithelial T cells were more prevalent in tumors with increased proliferation, indicating that the improved outcome is not due to indolent tumor cell behavior [33].

The low neoantigen load, HR-proficient tumors exhibited significantly lower numbers of CD3+ and CD8+ TILs, as well as lower expression of the inhibitory immune checkpoint modulators, PD-1 and PD-L1, compared to BRCA1/2-mutated tumors. This observation is consistent with the hypothesis that an elevated neoantigen load leads to an increased number of TILs that are counterbalanced by overexpression of immune checkpoint modulators [34].

The relative contribution of platinum versus taxane to the immunostimulatory effects seen in our study is an open question because nearly all patients received the combination therapy. Experimental studies suggest that cisplatin does not induce immunogenic cell death (ICD) but carboplatin and docetaxel produce partial features of ICD. Chemotherapy can also acti- vate the host immune system by several other mechanisms such as increased presentation of neoantigens. The association of antitumor immune response (intraepithelial T cells) with prolonged survival, and viceversa the association of immune escape mechanisms with poor survival, suggest that ovarian cancers are intrinsically immunogenic [35].

The presence or absence of T cells in solid tumors is a key limiting factor for cancer immunotherapy [23] and the presence of CD8+ T cells at the invasive margin was predictive of response.

\section{CONCLUSION}

The high number of tumor-infiltrating lymphocytes in post-surgery samples was significantly associated with higher rates of complete pathological response and better prognosis. So, the incorporation of immunotherapies to postchemotherapy treatment options could be of benefit for prolonged disease control in patients with advanced HGSC. It is convenient to carry out further and multicentric studies to validate these results.

Conflict of Interest: JAFV and AG-P are shareholders in Biomedica Molecular Medicine SL. LT-F and GP-V are employees of Biomedica Molecular Medicine SL. The other authors declare no competing interests.

\section{LIST OF ABBREVIATIONS}

NACT: Neoadjuvant chemotherapy; HGSC: High-grade serous ovarian carcinoma; TILs: Tumor-infiltrating lymphocytes; EOC: Epithelial ovarian cancer; OC: Ovarian cancer; AUC: Area under the curve; Tregs: Regulatory T cell; OS: Overall survival; FIGO: International Federation of Gynecologists and Obstetricians; Interval debulking surgery (IDS): PDS: Primary debulking surgery; CRS: Score response system; FFPE: Formalin-fixed paraffin embedded; ICCR: International collaboration on cancer reporting; NA: Not applicable; pCR: pathological complete response; OR: Odds ratio; HR: Hazard ratio; ICD: Immunogenic cell death. FOXP3: Forkhead box P3, CD4: Cluster differentiation factor 4

\section{References}

1. Stegel R, Naishadham D, Jemal A (2013) Cancer statistics. CA Cancer J Clin 63(1): 11-30

2. EUCAN Cancer Factsheets: Ovary [Internet] http://eco.iarc. fr/eucan/CancerOne.aspx?Cancer=27\&Gender=2 December 20, 2017 date last accessed 
3. Kurman RJ (2013) Origin and molecular pathogenesis of ovarian high-grade serous carcinoma. Ann Oncol 24 (Suppl 10): 16-21

4. Winter WE 3rd, Maxwell GL, Tian C, Carlson JW, Ozols RF, Rose PG, et al. (2007) Prognostic factors for stage III epithelial ovarian cancer. J Clin Oncol 25(24): 3621-3627

5. du Bois A, Quinn M, Thigpen T, Vermorken J, Avall-Lundgvist E, Bookman M, et al. (2005) Consensus statements on the management of ovarian cancer: final document of the 3rd International Gynecologic Cancer Intergroup Ovarian Cancer Consensus Conference (GCIG OCCC 2004). Ann Oncol 16 (Suppl 8): viii7-viii12

6. Chi DS, Musa F, Dao F, Zivanovic O, Sonoda Y, Leitao MM, et al. (2012) An analysis of patients with bulky advanced stage ovarian, tubal, and peritoneal carcinoma treated with primary debulking surgery (PDS) during an identical time period as the randomized EORTC-NCIC trial of PDS vs neoadjuvant chemotherapy (NACT). Gynecol Oncol 124(1):10-14

7. Preston CC, Maurer MJ, Oberg AL, Visscher DW, Kalli KR, Hartmann LC, et al. (2013) The ratios of CD8+ T cells to CD4+CD25+FOXP3+ and FOXP3-T cells correlate with poor clinical outcome in human serous ovarian cancer. PLoS One 8(11): e80063

8. Zhang L, Conejo-Garcia JR, Katsaros D, Gimotty PA, Mossobrio M, Regnani G, et al. (2003) Intratumoral T cells, recurrence, and survival in epitelial ovarian cancer. $\mathrm{N}$ Engl J Med 348(3): 203-213

9. Mony JT, Zhang L, Ma T, Grabosch S, Tirodkar TS, Brozick J, et al. (2015) Anti-PD-L1 prolongs survival and triggers $T$ cell but not humoral anti-tumor immune responses in a human MUC1-expressing preclinical ovarian cancer model. Cancer Immunol Immunother 64(9):1095-108

10. Sato E, Olson SH, Ahn J, Bundy B, Nishikawa H, Qian F, et al. (2005) Intraepithelial CD8+ tumor-infiltrating lymphocytes and a high CD8+/regulatory $T$ cell ratio are associated with favorable prognosis in ovarian cancer. Proc Natl Acad Sci USA 102(51):18538-18543

11. Coleman S, Clayton A, Mason MD, Jasani B, Adams M,Tabi Z (2005) Recovery of CD8+ T cell function during systemic chemotherapy in advanced ovarian cancer. Cancer Res 65(15):7000-7006
12. Webb JR, Milne K, Nelson BH (2015) PD-1 and CD 103 are widely coexpressed on prognostically favorable intraepithelial CD8 T cells in Human Ovarian Cancer. Cancer Immunol Res 3(8):926-935

13. M Stumpf, A Hasenburg, M-O Riener, U Jutting, $C$ Wang, Y Shen, et. al. (2009) Intraepithelial CD8-positive T lymphocytes predict survival for patients with serous stage III ovarian carcinomas: relevance of clonal of T lymphocytes. Br J Cancer 101(9):1513-1521

14. Preston CC, Goode EL, Hartmann LC, Kalli KR, Knutson KL (2011) Immunity and immune suppression in ovarian cancer. Immunotherapy 3(4): 539-556

15. Curiel TJ, Coukos G, Zou L, Alvarez X, Cheng P, Mottram P, et al. (2004) Specific recruitment of regulatory $T$ cells in ovarian carcinoma fosters immune privilege and predicts reduced survival. Nat Med 10(9):942-949

16. Maine CJ, Aziz NH, Chatterjee J, Hayford C, Brewig N, Whilding L, et al. (2014) Programmed death ligand-1 over-expression correlates with malignancy and contributes to immune regulation in ovarian cancer. Cancer Immunol Immunother 63(3): 215-224

17. Abiko K, Mandai M, Hamanishi J, Yoshioka Y, Matsumura N, Babu T, et al. (2013) PD-L1 on tumor cells is induced in ascitis and promotes peritoneal dissemination of Ovarian Cancer through CTL Dysfunction. Clin Cancer Res 19(6):1363-1374

18. Hamanishi J, Mandai M, Iwasaki M, Okazaki T, Tanaka Y, Yamaguchi K, et al. (2007) Programmed cell death 1 ligand 1 and tumor-infiltrating CD8+ T lymphocytes are prognostic factors of human ovarian cancer. Proc Natl Acad Sci USA 104(9):3360-3365

19. deLeeuw RJ, Kroeger D, Kost SE, Chang PP, Webb JR, Nelson BH (2015) CD25 Identifies a subset of CD4+FOXP3-TIL that are exhausted yet prognostically favorable in human. Ovarian Cancer 3(3): 245-253

20. Maine CJ, Aziz NH, Chatterjee J, Hayford C, Brewig N, Whilding $L$, et al. Programmed death ligand-1 over-expression correlates with malignancy and contributes to immune regulation in ovarian cancer. Cancer Immunol Immunother 63: 215-224

21. Baert T, Timmerman D, Vergote I, Coosemans A (2015) Immunological parameters as a new lead in the diagnosis of 
ovarian cancer. Facts Views Vis Obgyn 7(1):67-72

22. Harris M (2004) Monoclonal antibodies as therapeutic agents for cancer. Lancet Oncol 5(5):292-302

23. Hayashi K, Yonamine K, Masuko-Hongo K, lida T, Yamamoto K, Nishioka K, et al. (1999) Clonal expansion of T cells that are specific for autologous ovarian tumor among tumor-infiltrating T cells in humans. Gynecol Oncol 74(1):8692

24. Santin AD, Bellone S, Ravaggi A, Pecorelli S, Cannon MJ, Parham GP (2000) Induction of ovarian tumor-specific CD8+cytotoxic T lymphocytes by acid-eluted peptide-pulsed autologous dendritic cells. Obstet Gynecol 96(3):422-430

25. Peoples GE, Schoof DD, Andrews JV, Goedegebuure PS, Eberlein TJ (1993) T-cell recognition of ovarian cancer. Surgery 114(2):227-234

26. Bohm S, Faruqi A, Said I, Lockley M, Brockbank E, Jeyarajah A, et al. (2015) Chemotherapy Response Score: Development and validation of a System to Quantify Histopathologic Response to Neoadjuvant Chemotherapy in Tubo-Ovarian High-Grade Serous Carcinoma. J Clin Oncol 33(22):2457-2463

27. Bohm S, Montfort A, Pearce OM, Topping J, Chakravarty P, Everitt GL, et al. (2016) Neoadjuvant Chemotherapy Modulates the Immune Microenvironment in Metastases of Tubo-Ovarian High-Grade Serous Carcinoma. Clin Cancer Res 22(12):3025-3036

28. Williams MA, Holmes BJ, Sun JC, Bevan MJ (2006) Developing and maintaining protective CD8+ memory $T$ cells, Immunol Rev 211:146-153
29. Janssen EM, Droin NM, Lemmens EE, Pinkoski MJ, Bensinger SJ, Ehst BD, et al. (2005) CD4+ T-cell help controls CD8+ T-cell memory via TRAIL- mediated activation-induced cell death. Nature 434(7029): 88-93

30. Protti MP, De Monte L, Di Lullo G (2014) Tumor antigen-specific CD4+ T cells in cancer immunity: from antigen identification to tumor prognosis and development of therapeutic strategies. Tissue Antigens 83(4): 237-246

31. Ophir E, Bobisse S, Coukos G, Harari A, Kandalaft LE (2016) Personalized approaches to active immunotherapy in cancer. Biochim Biophys Acta 1865(1):72-82

32. Bachmayr-Heyda A, Aust S, Heinze G, Polterauer S, Grimm C, Braicu El, et al. (2013) Prognostic impact of tumor infiltrating CD8+ T cells in association with cell proliferation in ovarian cancer patients- a study of the OVCAD consortium. BMC Cancer 13:422

33. Adams SF, Levine DA, Cadungog MG, Hammond R, Facciabene A, Olvera N, et al. (2009) Intraepithelial T cells and tumor proliferation: Impact on the benefit from surgical cytoreduction in advanced serous ovarian cancer. Cancer 115(13):2891-2902

34. Strickland KC, Howitt BE, Shukla SA, Rodig S, Ritterhouse LL, Liu JF, et al. (2016) Association and prognostic significance of BRCA1/2-mutation status with neoantigen load, number of tumor-infiltrating lymphocytes and expression of PD-1/PD-L1 in high grade serous ovarian cancer. Oncotarget 7(12):13587-13598

35. Kandalaft LE, Powell DJ Jr, Singh N, Coukos G (2011) Immunotherapy for Ovarian Cancer: What's next? J Clin Oncol 29(7): 925-933

Copyright: $\odot 2019$ Rojas K, et al. This is an open-access article distributed under the terms of the Creative Commons Attribution License, which permits unrestricted use, distribution, and reproduction in any medium, provided the original author and source are credited. 